\title{
Reversal of Cognitive Dysfunction by Total Removal of a Large Lateral Ventricle Meningioma: A Case Report with Neuropsychological Assessments
}

\author{
Daina Kashiwazaki ${ }^{a} \quad$ Akiko Takaiwa $^{a}$ Shoichi Nagai ${ }^{a}$ Naoki Akioka ${ }^{a}$

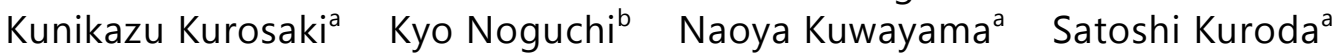 \\ Departments of ${ }^{a}$ Neurosurgery and ${ }^{b}$ Radiology, University of Toyama, Toyama, Japan
}

\section{Key Words}

Cognitive dysfunction · Neuropsychological assessments · Ventricle meningioma

\begin{abstract}
Although cognitive impairment is one of the major symptoms of ventricular tumors, few studies have reported the details of cognitive impairment before and after their surgical removal. The expected effects on cognitive function should also be considered when choosing a surgical approach. We report the case of a large lateral ventricle meningioma in which cognitive impairment was detected on detailed neuropsychological examinations. The tumor was totally removed through the right superior temporal gyrus. Postoperative neuropsychological assessment revealed the reversal of cognitive impairment. As cognitive impairment is complex and easily overlooked, it is important to precisely assess neuropsychological function in patients with large brain tumors.

(c) 2014 S. Karger AG, Basel
\end{abstract}

\section{Introduction}

Lateral ventricle meningiomas usually cause no significant clinical symptoms when they are small. As the tumors grow larger, however, they gradually elevate intracranial pressure and patients often present with visual field defects, limb weakness, and cognitive impairment such as memory disturbance and miscalculation. Surgical removal of large lateral ventricle meningiomas is still challenging. Surgical procedures including operative

Daina Kashiwazaki, MD

Department of Neurosurgery

University of Toyama

2630 Sugitani, Toyama 930-0194 (Japan)

E-Mail gktqx702@yahoo.co.jp 
approaches have been extensively debated in order to avoid postoperative complications [14]. Among these studies, only a few have discussed cognitive impairment in detail.

Here, we report the case of a large lateral ventricle meningioma associated with cognitive impairment. Precise neuropsychological examinations revealed that total removal combined with preoperative embolization achieved complete resolution of the cognitive impairment.

\section{Case Presentation}

A 66-year-old, right-handed man earned his living by cultivating agricultural products and cooking. His highest level of educational was high school graduation. He had no past medical history and had been incidentally diagnosed with a right ventricular meningioma 20 years earlier. He had no neurological symptoms and was conservatively followed up because the tumor diameter was about $1 \mathrm{~cm}$. However, the tumor had gradually increased in size on MRI during these 20 years. Finally, over the past year, his working performance had started to deteriorate. He experienced increasing difficulty in concentrating on various tasks (making errors while cooking, miscalculations, and aprosexia) and gradually exhibited inappropriate social behaviors (abusive words, violent manner, and juvenile behavior). His short-term memory also declined. Therefore, he was referred to our hospital for further examination. A standard neurological examination performed on admission revealed no definite abnormalities such as auditory agnosia, left hemispatial neglect, 2-point discrimination, callosal signs, optic ataxia, and astereognosis. Callosal signs such as auditory disconnection syndrome, extinction, and left-hand apraxia were absent. His visual field was kept intact on Goldmann visual field testing. MRI showed a large round mass in the trigone of the right lateral ventricle with a diameter of $5.2 \mathrm{~cm}$ and large perifocal edema around the ventricle limbic structure. The signal of the mass was low and high on T1- and T2-weighted images, respectively. The entire mass was strongly enhanced with Gd-DTPA (fig. 1). Cystic formation was observed lateral to the tumor. The midline structures were shifted to the left, and cerebral edema was observed in the white matter around the tumor. MR tractography could clearly localize the optic radiations around the tumor (fig. 2). Cerebral angiography showed that the posterior lateral choroidal artery was the main feeder of the tumor. Based on these findings, the tumor was diagnosed as meningioma. Then, cognitive function was precisely evaluated, using the Mini-Mental State Examination (MMSE), Standard Language Test of Aphasia (SLTA), and Repeatable Battery for the Assessment of Neuropsychological Status (RBANS). The scores on the MMSE and SLTA were within normal limits: 27 points and normal, respectively. On the other hand, RBANS revealed that the patient had marked impairment of attention, language, visuospatial/constructional abilities, and delayed memory function (fig. 3).

Prior to surgery, the lateral posterior choroidal artery was embolized via a microcatheter, using n-butyl-2-cyanoacrylate. MRI following embolization showed that the major part of the tumor was not further enhanced. Two days later, the patient underwent total tumor removal. A large temporoparietal craniotomy was performed, and the dura mater was opened. Under intraoperative navigation, the tumor was exposed through a 2-cm corticotomy on the superior temporal gyrus. The tumor was totally removed without any significant bleeding. Transfusion was not performed.

The postoperative course was uneventful, and the patient's motor and visual functions were intact. Moreover, a standard neurological examination performed after tumor removal revealed no abnormalities such as auditory agnosia, hemispatial neglect, 2-point discrimina- 
tion, callosal signs, optic ataxia, and astereognosis. Callosal signs such as disconnection syndrome, left-hand apraxia, and auditory extinction were also absent. His visual field was kept intact on Goldmann visual field testing. Total tumor removal was confirmed on followup MRI. Neuropsychological examinations were repeated 13 days after surgery. Results showed that all scores on RBANS significantly improved back to the normal range (fig. 3). Difficulty in concentrating on various tasks and the exhibiting of inappropriate social behaviors were completely resolved. He could perform various tasks (without making errors while cooking, miscalculation, and aprosexia) in his daily living after tumor removal. He was discharged and returned to his former position. A histopathological examination confirmed the diagnosis of fibrous meningioma. The mib-1 index was $2.3 \%$.

\section{Discussion}

Cognitive Impairment in Lateral Ventricle Tumors

Cognitive impairment is well known as a major symptom of lateral ventricular tumors and may be potentially complicated after their surgical removal [1-4]. Detailed neuropsychological evaluation would be essential in these cases because of its serious impact on patients' quality of life. However, there are few reports that precisely denote cognitive function in patients with lateral ventricle tumors. According to previous studies, the prevalence of preoperative cognitive impairment and personality changes is estimated at $10-20 \%$ in these patients [5]. However, this value may be much higher because Milligan and Meyer [6] reported that about half of patients had these symptoms when cognitive function tests were precisely performed by medical neurologists. Either the institutional practice of providing an objective pre- and postoperative neuropsychological assessment or a bias in reporting of cognitive impairment by family members may be responsible for the difference in the reported rates of preoperative cognitive impairment. This discrepancy strongly suggests that a full, detailed neuropsychological examination would be essential to accurately evaluate patients' cognitive function. It is known that involvement of periventricular limbic structures or hydrocephalus is the cause of cognitive impairment. In this case, large perifocal edema and cystic formation by the meningioma were the main causes of cognitive impairment. Patients with large edema or hydrocephalus should be examined by detailed neuropsychological examination.

The authors conducted the MMSE, SLTA, and RBANS before and after tumor removal to precisely evaluate cognitive impairment. The scores on the MMSE and SLTA were within normal limits, while the RBANS could clearly identify cognitive dysfunction in the present case. The RBANS consists of 12 subtests and yields index scores for 5 traits: attention (digit span, coding), language (picture naming, semantic fluency), visuospatial/constructional abilities (figure copy, line orientation), immediate memory (list learning, story memory), and delayed memory (list recall, list recognition, story recall, figure recall). Scores can be normalized for age, sex, ethnicity, and level of education, with a score of 100 and a standard deviation of 15 for the index group [7]. The RBANS includes 2 different test batteries for various cognitive domains without learning effects, being suitable to accurately assess neuropsychological changes before and after surgery. Each repeated test can be performed within about $30 \mathrm{~min}$ [8]. The RBANS is known to have the ability to detect a mild, potential decline of cognitive function more sensitively than other neuropsychological batteries. These observations strongly suggest that cognitive impairment is highly complex and may be overlooked by brief neuropsychological instruments such as the MMSE. Furthermore, although the SLTA result was within the normal range, language evaluated by the RBANS 
was impaired. This result suggests limitation of evaluation by a single neuropsychological test. Therefore, a detailed neuropsychological examination is warranted to accurately evaluate cognitive impairment in patients with lateral ventricle tumors. Preoperative neuropsychological assessment is especially crucial in these patients because surgical approaches to the lateral ventricle may potentially cause permanent cognitive dysfunction through injury to delicate areas. Based on this case, cognitive impairment due to the effects of a tumor may be reversible.

\section{Treatment Strategy for Lateral Ventricle Tumors}

In general, there are 3 main surgical approaches to remove lateral ventricle tumors: the superior parietal, transcallosal, and middle temporal gyrus approaches [6, 9-12]. The choice of surgical approach depends on tumor location, laterality, symptoms, feeders, and tumor size. In the present case, however, the authors did not select these approaches. Superior parietal approach has been reported to carry a higher inherent risk of cognitive impairment $[7,13,14]$. Instead, the authors approached the trigone of the lateral ventricle through the superior temporal gyrus of the non-dominant hemisphere. As a result, the tumor could be totally removed. The patient's visual field could be kept intact and neuropsychological function markedly improved after surgery. The authors chose this route because it provided the shortest trajectory to the tumor and had a lower risk of cognitive impairment. When deciding on the surgical approach, MR tractography was quite useful to avoid postoperative visual field disturbance by visualizing the optic radiation [15].

Furthermore, preoperative endovascular embolization was quite valuable to markedly reduce bleeding from the tumor during surgery. Enhanced MRI before and after embolization clearly showed the therapeutic effects. The posterior lateral choroidal artery, especially, cannot easily be accessed during surgery; thus, its preoperative embolization enabled us to remove it with minimal surgical risk.

In conclusion, pre- and postoperative precise neuropsychological examinations may identify the potential cognitive impairment and beneficial effects of surgery in patients with large lateral ventricle meningiomas. Prior to surgery, MR tractography and feeder embolization were quite useful to achieve total tumor removal with minimal surgical risks.

\section{Disclosure Statement}

The authors declare no conflict of interest associated with the manuscript.

\section{References}

1 Barrow DL, Dawson R: Surgical management of arteriovenous malformations in the region of the ventricular trigone. Neurosurgery 1994;35:1046-1054.

2 Jun CL, Nutik SL: Surgical approaches to intraventricular meningiomas of the trigone. Neurosurgery $1985 ; 16: 416-420$.

-3 Nagata S, Sasaki T: Lateral trans-sulcal approach to asymptomatic trigonal meningiomas with correlative microsurgical anatomy: technical case report. Neurosurgery 2005;56(2 suppl):E438.

4 Nishizaki T, Ikeda N, Nakano S, Okamura T, Abiko S: Occipital inter-hemispheric approach for lateral ventricular trigone meningioma. Acta Neurochir (Wien) 2009;151:1717-1721.

-5 Gokalp HZ, Yuceer N, Arasil E, Deda H, Attar A, Erdoğan A, Egemen N, Kanpolat Y: Tumours of the lateral ventricle. A retrospective review of 112 cases operated upon 1970-1997. Neurosurg Rev 1998;21:126-137.

6 Milligan BD, Meyer FB: Morbidity of transcallosal and transcortical approaches to lesions in and around the lateral and third ventricles: a single-institution experience. Neurosurgery 2010;67:1483-1496. 


\section{Case Reports in Neurology}

\begin{tabular}{l|l}
\hline Case Rep Neurol 2014;6:44-49 \\
\hline DOI: $10.1159 / 000358819$ & $\begin{array}{l}\text { (c) 2014 S. Karger AG, Basel } \\
\text { www.karger.com/crn }\end{array}$ \\
\hline
\end{tabular}

Kashiwazaki et al.: Reversal of Cognitive Dysfunction by Total Removal of a Large

Lateral Ventricle Meningioma: A Case Report with Neuropsychological Assessments

7 Randolph C, Tierney MC, Mohr E, Chase TN: The Repeatable Battery for the Assessment of

Neuropsychological Status (RBANS): preliminary clinical validity. J Clin Exp Neuropsychol 1998;20:310319.

8 Levin HS, Speirs PA: Acalculia, in Heilman K, Valenstein E: Clinical Neuropsychology. New York, Oxford University Press, 1985, pp 97-113.

9 Guidetti B, Delfini R, Gagliardi FM, Vagnozzi R: Meningiomas of the lateral ventricles. Clinical, neuroradiologic, and surgical considerations in 19 cases. Surg Neurol 1985;24:364-370.

10 Heilman KM, Gonzales Rothi LJ: Apraxia, in Heilman K, Valenstein E (eds): Clinical Neuropsychology. New York, Oxford University Press, 1985, pp 131-149.

11 Kempe LG, Blaylock R: Lateral-trigonal intraventricular tumors. A new operative approach. Acta Neurochir (Wien) 1976;35:233-242.

$\$ 12$ Mahaney KB, Abdulrauf SI: Anatomic relationship of the optic radiations to the atrium of the lateral ventricle: description of a novel entry point to the trigone. Neurosurgery 2008;63(4 suppl 2):195-202.

13 Fornari M, Savoiardo M, Morello G, Solero CL: Meningiomas of the lateral ventricles. Neuroradiological and surgical considerations in 18 cases. J Neurosurg 1981;54:64-74.

14 Takaiwa A, Hayashi N, Kuwayama N, Akioka N, Kubo M, Endo S: Changes in cognitive function during the 1year period following endarterectomy and stenting of patients with high-grade carotid artery stenosis. Acta Neurochir (Wien) 2009;151:1593-1600.

-15 Powell HW, Parker GJ, Alexander DC, Symms MR, Boulby PA, Wheeler-Kingshott CA, Barker GJ, Koepp MJ, Duncan JS: MR tractography predicts visual field defects following temporal lobe resection. Neurology 2005;65:596-599.

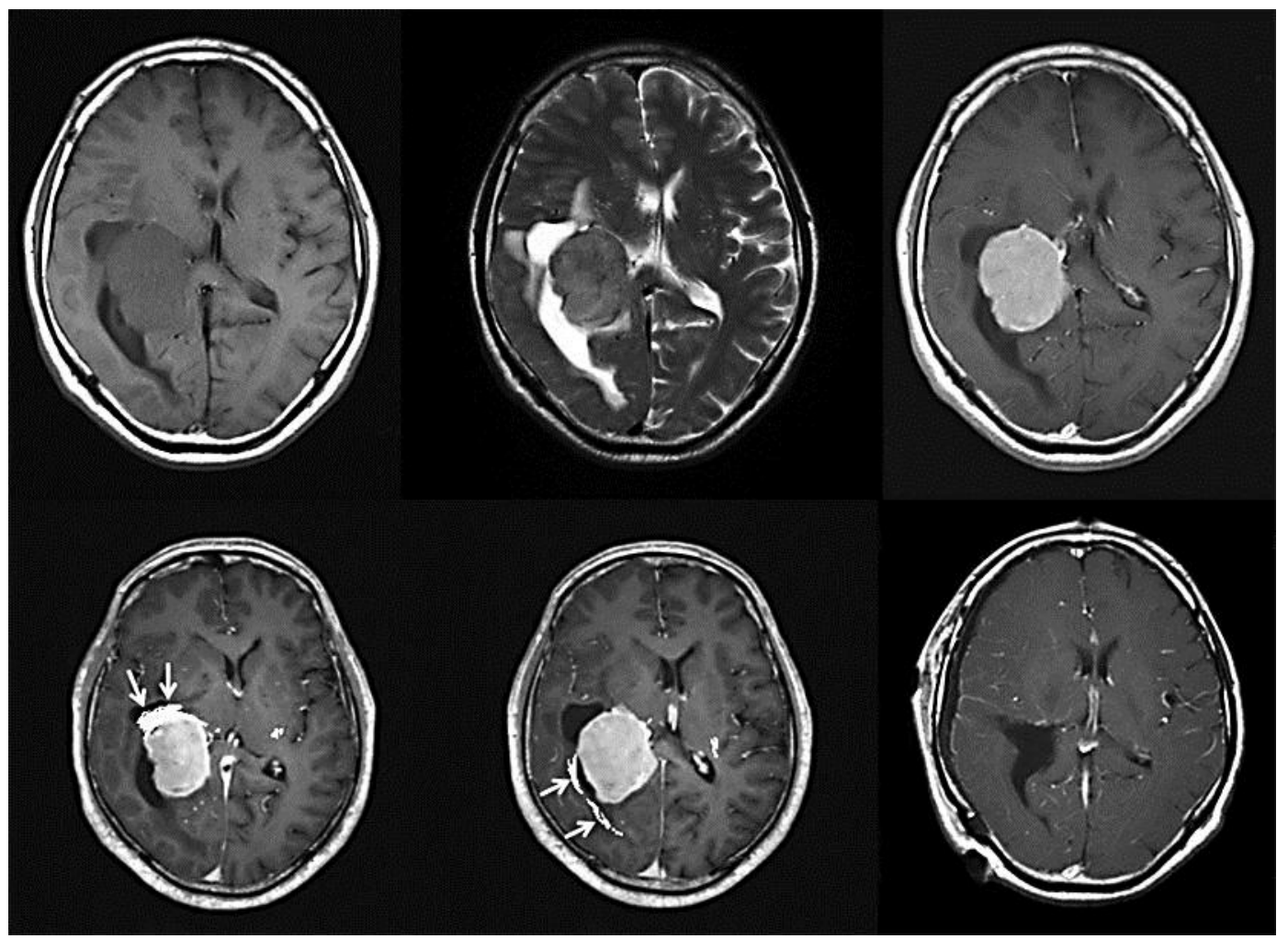

Fig. 1. MRI shows low intensity on T1-weighted images and high intensity on T2-weighted images, with strong high enhancement on contrast. Arrows indicate optic radiation. 


\section{Case Reports in Neurology}

Case Rep Neurol 2014;6:44-49

DOI: $10.1159 / 000358819$

Kashiwazaki et al.: Reversal of Cognitive Dysfunction by Total Removal of a Large

Lateral Ventricle Meningioma: A Case Report with Neuropsychological Assessment

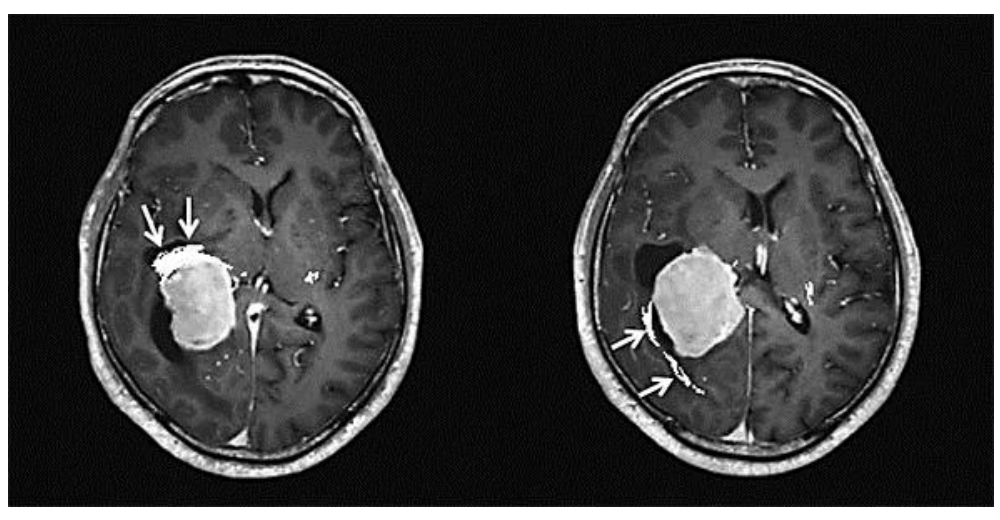

Fig. 2. Optic radiation tractography using MRI shows the visualized optic radiation that runs laterally to the tumor (arrows).

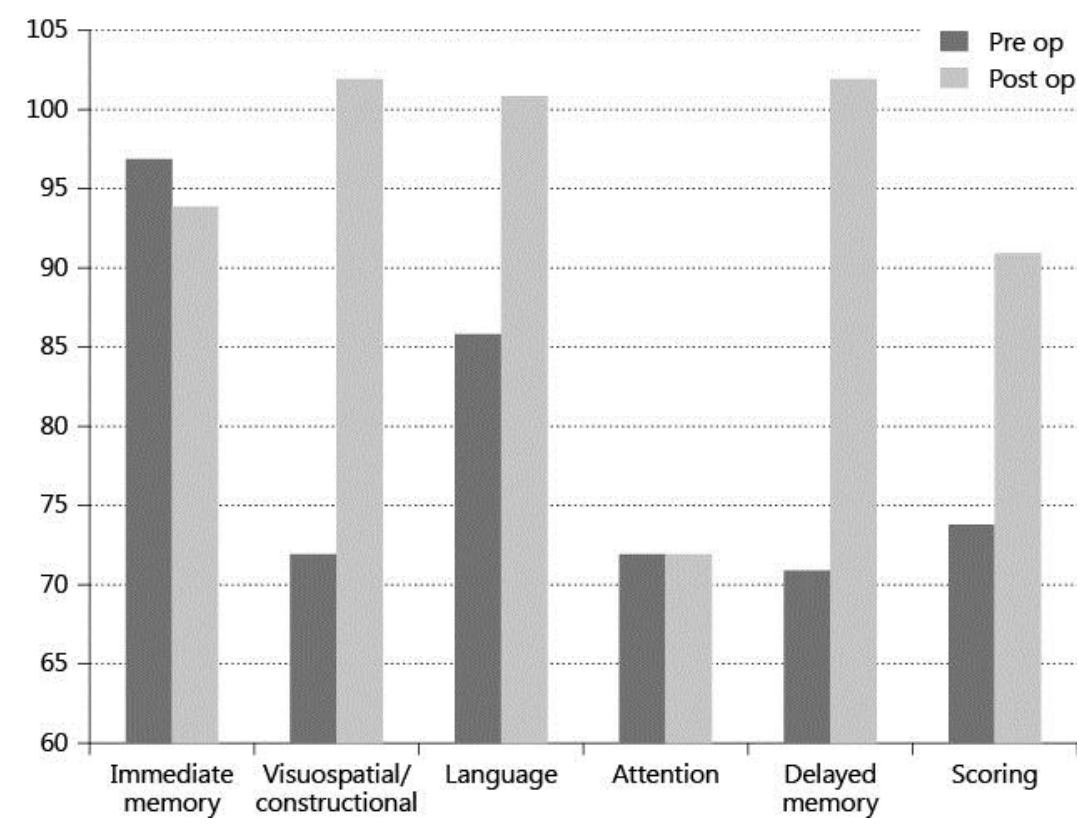

Fig. 3. Results of the tests included in the RBANS before and after tumor removal. 